\title{
Re: Ethylene Glycol Ingestion Treated Only With Fomepizole (Journal of Medical Toxicology: Volume 3, Number 3, September 2007; 125-128)
}

Velez et al's recent case report [1], describing the efficacy of fomepizole as the sole antidotal therapy for ethylene glycol toxicity, is pertinent to the practice of medical toxicology as there is now a mounting body of evidence highlighting the value of this treatment approach. Hemodialysis is not a benign procedure; hypotension, the most worrisome acute complication, is cited to occur in $25-55 \%$ of treatments [2]. Furthermore, the overall costeffectiveness must be considered when taking into account resource utilization. In a case report by Boyer et al [3], the cost of using fomepizole as a single therapy was compared against that of hemodialysis, with a $65 \%$ increased cost associated with the latter modality. When hemodialysis is used as the primary therapy, these patients concomitantly receive fomepizole at fourhour dosing intervals instead of every twelve hours; the antidote itself is also eliminated by dialysis, further increasing the financial burden.

Our toxicology consult service recently cared for a 45 -yearold female whose initial ethylene glycol level was $444 \mathrm{mg} / \mathrm{dL}$; she was also successfully treated with fomepizole alone without concomitant use of hemodialysis. Adjunctive therapy included thiamine, folic acid and pyridoxine. Her renal function and acid-base status remained normal throughout her hospital stay. Our case represents the $3^{\text {rd }}$ highest serum ethylene glycol concentration reported in the literature that was treated without hemodialysis, mirroring that of Velaz et al, as well as Aakervik et al [4], where the peak serum ethylene glycol concentrations were $706 \mathrm{mg} / \mathrm{dl}$ and $585 \mathrm{mg} / \mathrm{dl}$, respectively. Our case further supports the use of fomepizole alone for severe ethylene glycol intoxication in cases where the acid-base status and renal function are normal; such an approach may obviate the need for hemodialysis, with its known complications, as well as mitigate the financial costs clearly associated with aggressive renal interventions [5].
Mathew George, MD

Nadeem Al-Duaij, MD

Melisa Lai Becker, MD

Michele Burns Ewald, MD

Harvard Medical Toxicology Fellowship

Regional Center for Poison Control \& Prevention serving

Massachusetts \& Rhode Island

Children's Hospital Boston

300 Longwood Avenue

Boston, MA 02115

Email: Mathew.george@childrens.harvard.edu

Phone: 617.355.6609

The authors do not have any conflicts of interest. This data has not been presented elsewhere.

\section{REFERENCES}

1. Velez, LI, Shepherd G, Lee YK, Keyes DC. Ethylene glycol ingestion treated only with fomepizole. Journal of Medical Toxicology. 2007; 3 (3): 125-128.

2. Bregman H, Daugirdas JT, Ing TS. Complications during hemodialsysis. In: Handbook of Dialysis. Daugirdas JT, Ing TS (Eds), Little Brown, New York, 1994, p. 149.

3. Boyer EW, Mejia M, Woolf A, Shannon M. Severe ethylene glycol ingestion treated without hemodialysis. Pediatrics. 2001; 107:172-173.

4. Aakervik O, Svendsen J, Jacobsen D. [Severe ethylene glycol poisoning treated with fomepizole (4-methylpyrazole)]. Tidsskr Nor Laegeforen. 2002; 122 (25): 2444-2446.

5 . Green R. The management of severe toxic alcohol ingestions at a tertiary care center after the introduction of fomepizole. Am J Emerg Med. 2007; 25:799-803. 